\title{
Perturbations of the harmonic map equation
}

\author{
T. Kappeler
}

\begin{abstract}
We consider perturbations of the harmonic map equation in the case where the source and target manifolds are closed Riemannian manifolds and the latter is in addition of nonpositive sectional curvature. For any semilinear and, under some extra conditions, quasilinear perturbation, the space of classical solutions within a homotopy class is proved to be compact. For generic perturbations the set of solutions is finite and we present a count of this set. An important ingredient for our analysis is a new inequality for maps in a given homotopy class which can be viewed as a version of the Poincaré inequality for such maps.
\end{abstract}

\section{Introduction}

In this paper we report on joint work with S. Kuksin and V. Schroeder as well as J. Latschev on semilinear and quasilinear perturbations of the harmonic map equation $\tau(u)=0$ following the expositions in [KKS1],[KKS2],[KL].

The harmonic map equation is an equation for maps $u: M \rightarrow M^{\prime}$ between Riemannian manifolds, defined as the Euler-Lagrange equation for the energy functional

$$
E(u):=\int_{M} e(u)(x) d \operatorname{vol}(x)
$$

where $e(u)(x)$ denotes the energy density,

$$
e(u)(x):=\frac{1}{2} g^{i j}(x)\left\langle\frac{\partial u}{\partial x^{i}}, \frac{\partial u}{\partial x^{j}}\right\rangle .
$$

Here $g^{i j}(x)$ is the inverse of the (smooth) metric tensor on $M, d \operatorname{vol}(\mathrm{x})$ the corresponding volume element, and $\langle\cdot, \cdot\rangle$ the scalar product in $T M^{\prime}$. In local coordinates for $M$ and $M^{\prime}$, the operator $\tau$ can be written as the Laplace-Beltrami operator, perturbed by terms which are quadratic in the derivatives of $u$.

If not stated otherwise we assume throughout the paper that $M$ and $M^{\prime}$ are closed.

Supported in part by the European Research Training Network HPRN-CT-1999-00118. 
Our aim is to consider perturbations of the equation $\tau(u)=0$ by a semilinear term $F(x, u(x))$ with $F$ being a $x$-dependent vector field on $M^{\prime}$, and by terms linear in the derivatives of $u$ and of sufficiently small size of the form

$$
L(x, u(x)) u_{*}(G(x, u(x)))
$$

where $L(x, u(x))$ is a linear operator on $T_{u(x)} M^{\prime}, u_{*}$ the differential of $u$, and $G$ a $y$-dependent vector field on $M, G(x, u(x)) \in T_{x} M$. More precisely we want to study the set of solutions $u: M \rightarrow M^{\prime}$ of

$$
\tau(u)(x)+F(x, u(x))+L(x, u(x)) u_{*}(G(x, u(x)))=0
$$

in a given homotopy class $\zeta$ of $C^{1}$-maps $u: M \rightarrow M^{\prime}$,

$$
u \in \zeta
$$

Note that (0.1) is not necessarily of variational form. The main assumption we impose is that

$$
M^{\prime} \text { has nonpositive sectional curvature . }
$$

Unless otherwise stated this assumption will be made throughout the paper. Given $F, G$ and $L$ of class $C^{k}$ with $k \geq 2$, denote by $S_{F, G, L} \equiv S_{F, G, L}^{(k)}$ the set of all $C^{k+1}$ solutions of (0.1). In the case $L=0$ and $G=0$ we simply write $S_{F}$ instead of $S_{F, 0,0}$. We note that by the regularity theory of elliptic equations, given $F$ of class $C^{k}$, any $C^{3}$-solution $v \in S_{F}^{(2)}$ is $C^{k+1}$-smooth, i.e.

$$
S_{F}^{(2)}=S_{F}^{(k)}
$$

Similarly, if $\operatorname{dim} M \leq 3$ and $F, G$ and $L$ are of class $C^{k}$, one has $S_{F, G, L}^{(2)}=S_{F, G, L}^{(k)}$. It follows from [ES], [Ha] and [SY] that for any homotopy class $\zeta$, the set $S_{0} \cap \zeta$ of harmonic maps in $\zeta$ is a nonempty, connected and compact subset of $C^{\infty}\left(M, M^{\prime}\right)$. Our aim is to investigate if the solution set $S_{F, G, L}^{(k)} \cap \zeta$ has similar properties.

\section{Compactness}

First we prove that for any $F, G, L$ of class $C^{k}$ with $k \geq 2, S_{F} \cap \zeta$ is compact in the $C^{k+1}$ topology and, if $\operatorname{dim} M \leq 3, S_{F, G, L} \cap \zeta$ is compact in the $C^{k+1}$ topology for $G$ and $L$ of sufficiently small size,

$$
\max _{\substack{x \in M \\ y \in M^{\prime}}}(\|L(x, y)\| \cdot\|G(x, y)\|) \leq c_{*}
$$

where $c_{*}>0$ is a constant which only depends on $\zeta$ and the manifolds $M$ and $M^{\prime}$. Here $\|L(x, y)\|$ denotes the operator norm of $L(x, y): T_{y} M^{\prime} \rightarrow T_{y} M^{\prime}$ and $\|G(x, y)\|$ is the norm of $G(x, y)$ in $T_{x} M$.

In fact we prove slightly stronger results. To state them we first need to introduce some more notation. Let us denote by $\mathcal{C}^{k}$ the space of $C^{k}$-maps from $M$ to $M^{\prime}$, by $\mathcal{F}^{(k)}$ the vector space of $x$-dependent vector fields $F(x, y)$ on $M^{\prime}$ of class $C^{k}$ in $x$ 
and $y$, by $\mathcal{G}^{(k)}$ the vector space of $y$-dependent vector fields $G(x, y)$ on $M$ of class $C^{k}$ in $x$ and $y$ and by $\mathcal{L}^{(k)}$ the vector space of linear operators $L(x, y)$ on $T_{y} M^{\prime}$ of class $C^{k}$ in $x$ and $y$. Let

$$
\mathcal{M}_{\zeta}^{(k)}:=\left\{(u, F) \mid F \in \mathcal{F}^{(k)} ; u \in S_{F} \cap \zeta\right\}
$$

considered as a subset of $\mathcal{C}^{k+1} \times \mathcal{F}^{(k)}$ and, for any $c_{*}>0$

$$
\begin{aligned}
\mathcal{N}_{\zeta, c_{*}}^{(k)}:=\{(u, F, G, L) \mid & (F, G, L) \in \mathcal{F}^{(k)} \times \mathcal{G}^{(k)} \times \mathcal{L}^{(k)} ; u \in S_{F, G, L} \cap \zeta ; \\
& \left.\max _{x, y}\|L(x, y)\| \cdot\|G(x, y)\|<c_{*}\right\}
\end{aligned}
$$

considered as a subset of $\mathcal{C}^{k+1} \times \mathcal{F}^{(k)} \times \mathcal{G}^{(k)} \times \mathcal{L}^{(k)}$. By $\pi$ we denote the natural projections

$$
\pi: \mathcal{M}_{\zeta}^{(k)} \rightarrow \mathcal{F}^{(k)} \quad \text { or } \pi: \mathcal{N}_{\zeta}^{(k)} \rightarrow \mathcal{F}^{(k)} \times \mathcal{G}^{(k)} \times \mathcal{L}^{(k)}
$$

Recall that a continuous map between topological spaces is called proper if the preimage of any compact set is compact.

The main results in [KKS1] are the following ones:

Theorem 1.1 Let $M$ and $M^{\prime}$ be closed Riemannian manifolds with $M^{\prime}$ having nonpositive sectional curvature and $\zeta$ be a homotopy class of $C^{1}$-maps from $M$ to $M^{\prime}$. Then for any $k \geq 2$, the projection $\pi: \mathcal{M}_{\zeta}^{(k)} \rightarrow \mathcal{F}^{(k)}$ is proper.

Theorem 1.2 Let $M$ and $M^{\prime}$ be closed Riemannian manifolds with $M^{\prime}$ having nonpositive sectional curvature and $\zeta$ be a homotopy class of $C^{1}$-maps from $M$ to $M^{\prime}$. Assume that $k \geq 2$ and $\operatorname{dim} M \leq 3$. Then there exists $c_{*}>0$ such that $\pi: \mathcal{N}_{\zeta, c_{*}}^{(k)} \rightarrow \mathcal{F}^{(k)} \times \mathcal{G}^{(k)} \times \mathcal{L}^{(k)}$ is proper.

In particular, Theorem 1.1 contains the following extension of a result due to Schoen-Yau for harmonic maps [SY] (cf also Hartman [Ha]) concerning the compactness of the space of harmonic maps within a homotopy class.

Corollary 1.3 Let $F \in \mathcal{F}^{(k)}$ with $k \geq 2$. Then $S_{F} \cap \zeta$ is compact in the $C^{k+1}$. topology.

We note that a corollary of Theorem 1.2 similar to Corollary 1.3 holds.

Simple examples show that the stated result of Corollary 1.3 does not need to hold if $M^{\prime}$ is not of nonpositive sectional curvature and the statement of Theorem 1.2 is not necessarily true if the perturbation is not affine in the differential $u_{*}$ or the part which is linear in $u_{*}$ is not sufficiently small.

We remark that no efforts have been made to see if Theorem 1.1 and Theorem 1.2 hold for $k$ smaller than two. Moreover, most likely Theorem 1.2 holds for manifolds $M$ of arbitrary dimension.

Our results are similar in flavour to the compactness results due to Kuksin [Ku] for double periodic solutions of quasilinear Cauchy-Riemann equations which originated in a compactness result of Gromov [Gr] for $J$-holomorphic curves. In future work we plan to establish similar results for other important nonlinear elliptic equations. 
The main ingredient of the proof of Theorem 1.1 and Theorem 1.2 in [KKS1] is an apriori estimate for the energy $E(u)$ for a solution $u$ of $(0.1)$ in a given homotopy class $\zeta$ : As a first step we introduce canonical distance functions $N_{p}(u, v)(p \geq 1)$ between two maps $u, v: M \rightarrow M^{\prime}$ in $\zeta$ and prove that if $u, v$ are of class $C^{3}$ the energy $E(u)$ can be bounded by

$$
E(u) \leq\|F\|_{C^{0}} N_{1}(u, v)+\sqrt{2}\|G\|_{C^{0}} E(u)^{1 / 2} N_{2}(u, v)+E(v) .
$$

Here $N_{p}(u, v)$ is defined by

$$
N_{p}(u, v):=\inf \left\{N_{p}(H) \mid H \text { is a } C^{1} \text {-homotopy between } u \text { and } v\right\}
$$

with

$$
N_{p}(H):=\left(\int_{M}\left(\int_{0}^{1}\left\|\frac{d}{d s} H_{s}(x)\right\| d s\right)^{p} d \operatorname{vol}(x)\right)^{1 / p} .
$$

In a second step we show that $N_{2}(v, u)$ can be bounded in terms of $E(u)$ and $E(v)$ :

Theorem 1.4 Let $M$ and $M^{\prime}$ be closed Riemannian manifolds with $M^{\prime}$ having nonpositive sectional curvature and $\zeta$ be a homotopy class of $C^{1}$-maps from $M$ to $M^{\prime}$. Then there exists a constant $C>0$ such that for any $u, v \in \zeta$

$$
N_{2}(u, v) \leq C\left(E(u)^{1 / 2}+E(v)^{1 / 2}+1\right) .
$$

Our proof of Theorem 1.4 uses in an essential way that $M^{\prime}$ has nonpositive sectional curvature. The main ingredient of the proof is a version of the apriori estimate (1.1) for maps from a metric graph to $M^{\prime}$ which we discuss in the subsequent section.

In [KKS2] we prove a sharper version of Theorem 1.4: In the case where $M^{\prime}$ has negative sectional curvature there is a constant $C^{\prime}>0$ so that for any homotopic maps $u, v: M \rightarrow M^{\prime}$ inequality (1.1) holds with $C$ replaced by $C^{\prime}$.

Estimate (1.1) is a new inequality which can be viewed as a version of the Poincaré inequality for maps between manifolds and is of independent interest. It also has the flavour of a quadratic isoperimetric inequality. We illustrate this by considering the case when $M=S^{1}$. Viewing $E(u)^{1 / 2}$ as a measure for the length of $u$, inequality (1.1) says that there exists a homotopy such that the area of the cylinder given by the homotopy can be bounded in terms of the square of the length of its boundary. Here the area of the cylinder is measured in terms of its $L_{2}$-averaged "length" $N_{2}(u, v)$ and the length of its boundary by $E(u)^{1 / 2}+E(v)^{1 / 2}$. We recall that for a Hadamard space $X$ the following isoperimetric inequality holds: given any simple, closed curve $\gamma$ in $X$ of length $L$, there exists a disc $D$ with $\partial D=\gamma$ so that area $(D) \leq \pi L^{2}$.

\section{Short homotopies between graphs}

Let $M^{\prime}=X^{\prime} / \Gamma$ be a compact Riemannian manifold with nonpositive sectional curvature. In this section we show that two homotopic maps from a graph into $M^{\prime}$ can be joined by a short homotopy. 
Let $G$ be a finite metric graph, i.e. a finite graph, where every edge has some positive length ( $\mathrm{cf}[\mathrm{BH}]$ I.1.9). We also assume for simplicity that $G$ has no terminals, i.e. that every edge is incident to at least two edges. A map $u: G \rightarrow M^{\prime}$ is called differentiable, if the restriction of $u$ to every edge is differentiable. In an obvious way one defines the length $L(u)$ of a differentiable map $u: G \rightarrow M^{\prime}$ by summing up the lengths of the restriction of $u$ to any of the edges of $G$. The following result is the main ingredient in the proof of Theorem 1.4.

Theorem 2.1 Let $\zeta$ be a homotopy class of $C^{1}$-maps from $G$ to $M^{\prime}$. Then there is a constant $C_{0}>0$ such that for any $u, v \in \zeta$ there exists a homotopy $H: G \times[0,1] \rightarrow$ $M^{\prime}$ from $v$ to $u$, with the property that for any point $z \in G$,

$$
\ell_{H}(z) \leq C_{0}(L(u)+L(v)+1) .
$$

The constant $C_{0}$ does not depend on the choice of the metric on $G$. Here $\ell_{H}(z)$ denotes the length of the curve $s \mapsto H(z, s)$.

We outline the proof in the special case where $G$ is a circle with a given metric, the homotopy class $\zeta$ is nontrivial, and the sectional curvature of $M^{\prime}$ is strictly negative. Denote by $T \subset \mathbb{R}$ an interval of the same length as $G$ and by $p^{+}, p^{-}$its two endpoints. Let $\varphi: T \rightarrow G$ be the canonical map identifying $p^{+}$and $p^{-}$, choose $t_{0}=p^{+} \in T$ as a basepoint and let $H^{G}: G \times[0,1] \rightarrow M^{\prime}$ be a given homotopy from $v$ to $u$. Consider the map $H^{T}: T \times[0,1] \rightarrow M^{\prime}$ defined by $H^{T}(t, s)=H^{G}(\varphi(t), s)$ which can be lifted to a map $\bar{H}^{T}: T \times[0,1] \rightarrow X^{\prime}$. Since $H^{T}\left(p^{+}, s\right)=H^{T}\left(p^{-}, s\right)$ for any $0 \leq s \leq 1$ and $\Gamma$ acts discretely, there is a deck transformation $\gamma \in \Gamma$ so that $\gamma \bar{H}^{T}\left(p^{+}, s\right)=\bar{H}^{T}\left(p^{-}, s\right)$ for any $0 \leq s \leq 1$. Furthermore $\gamma$ is not the trivial element, since $\zeta$ is nontrivial. Denote by $d_{\gamma}$ the displacement function given by $d_{\gamma}(x):=d(x, \gamma x)$ where $d$ is the distance function on $X^{\prime}$. As, by assumption, the curvature is strictly negative, $\gamma$ translates a unique geodesic which coincides as a set with

$$
\operatorname{MIN}(\gamma):=\left\{x \in X^{\prime} \mid d_{\gamma}(x)=\inf _{y \in X^{\prime}} d_{\gamma}(y)\right\}
$$

(cf section 6 in [BGS]). Using the convexity of the distance function on the Hadamard space $X^{\prime}$ one can prove - see [KKS1], [BGS]

$$
d\left(\bar{H}^{T}\left(t_{0}, 1\right), \operatorname{MIN}(\gamma)\right) \leq \varrho d_{\gamma}\left(\bar{H}^{T}\left(t_{0}, 1\right)\right)+1 \leq \varrho L(u)+1
$$

for some $\varrho>0$, only depending on $\zeta$ and $M^{\prime}$, and similarly,

$$
d\left(\bar{H}^{T}\left(t_{0}, 0\right), \operatorname{MIN}(\gamma)\right) \leq \varrho L(v)+1
$$

Let $\pi_{\mathrm{MIN}(\gamma)}: X^{\prime} \rightarrow \operatorname{MIN}(\gamma)$ be the metric projection onto $\operatorname{MIN}(\gamma)$. The cyclic group $\langle\gamma\rangle$ operates with compact quotient on the geodesic $\operatorname{MIN}(\gamma)$ - see [KKS1], [CS]. Thus there exists $m \in \mathbb{Z}$ such that for $\alpha=\gamma^{m}$

$$
d\left(\alpha \pi_{\mathrm{MIN}(\gamma)} \bar{H}^{T}\left(t_{0}, 1\right), \pi_{\mathrm{MIN}(\gamma)} \bar{H}^{T}\left(t_{0}, 0\right)\right) \leq C
$$


where $C$ is the minimum of $d_{\gamma}$ which depends only on the homotopy class of $\zeta$. With the help of $\alpha$ we define a new homotopy $\hat{H}^{T}: T \times[0,1] \rightarrow X^{\prime}$ by $\hat{H}^{T}(t, s)=c_{t}(s)$ where for any $t \in T, c_{t}:[0,1] \rightarrow X^{\prime}$ is the geodesic from $\bar{H}^{T}(t, 0)$ to $\alpha \bar{H}^{T}(t, 1)$. Since $\alpha$ commutes with $\gamma$ it is easily checked that for any $0 \leq s \leq 1, \gamma \hat{H}^{T}\left(p^{+}, s\right)=$ $\hat{H}^{T}\left(p^{-}, s\right)$. Hence $\hat{H}^{T}$ induces a homotopy $H$ from $v$ to $u$. By $(2.1),(2.2)$ and $(2.3)$ we estimate

$$
\begin{aligned}
& d\left(\hat{H}^{T}\left(t_{0}, 1\right), \hat{H}^{T}\left(t_{0}, 0\right)\right) \\
& \leq d\left(\hat{H}^{T}\left(t_{0}, 1\right), \alpha \pi_{\mathrm{MIN}(\gamma)} \bar{H}^{T}\left(t_{0}, 1\right)\right)+d\left(\alpha \pi_{\mathrm{MIN}(\gamma)} \bar{H}^{T}\left(t_{0}, 1\right), \pi_{\mathrm{MIN}(\gamma)} \bar{H}^{T}\left(t_{0}, 0\right)\right) \\
& +d\left(\pi_{\mathrm{MIN}(\gamma)} \bar{H}^{T}\left(t_{0}, 0\right), \bar{H}^{T}\left(t_{0}, 0\right)\right) \\
& \leq(\varrho L(v)+1)+C+(\varrho L(u)+1) .
\end{aligned}
$$

Let $H: G \times[0,1] \rightarrow M^{\prime}$ be defined by composing $\hat{H}^{T}$ with the projection $X^{\prime} \rightarrow M^{\prime}$. Then the above inequality implies

$$
\ell_{H}\left(z_{0}\right) \leq C^{\prime}(L(u)+L(v)+1)
$$

for $z_{0}=\varphi\left(t_{0}\right)$ and $C^{\prime}$ is given by $C^{\prime}:=C+2+\varrho$ which is independent of the metric on $G$. Using the triangle inequality we obtain

$$
\ell_{H}(z) \leq\left(C^{\prime}+1\right)(L(u)+L(v)+1)
$$

for an arbitrary point $z \in G$. The argument in the general case is essentially the same. The interval $T$ has to be replaced by a suitable metric tree, $\operatorname{MIN}(\gamma)$ by $\operatorname{MIN}(f)$ for a suitable function $f=\sum_{i=1}^{m} d_{\gamma_{i}}$ and the group $\langle\gamma\rangle$ by the centralizer $Z\left(\gamma_{1}, \ldots \gamma_{m}\right)$ - see $[\mathrm{KKS} 1]$ for details.

In the case $M^{\prime}$ has strictly negative sectional curvature Theorem 2.1 can be improved. Denote by $N(u, v)$ the following distance between two homotopic $C^{1}$-maps $u, v: G \rightarrow M^{\prime}$,

$$
N(u, v):=\inf _{H}\left\{\sup _{z \in G} \ell_{H}(z)\right\}
$$

where the infimum is taken over all $C^{1}$-homotopies $H: G \times[0,1] \rightarrow M^{\prime}$ between $u$ and $v$ and $\ell_{H}(x)$ is the length of the curve $s \mapsto H(x, s)$. In [KKS2] we show the following estimate.

Theorem 2.2 Let $\kappa>0$ and $\varrho>0$ be given. Then for any Riemannian manifold $M^{\prime}$ with sectional curvature bounded from above by $-\kappa<0$ and injectivity radius bounded from below by $\varrho>0$, but not necessarily compact, for any finite metric graph $G$ and for any homotopic $C^{1}$ maps $u, v: G \rightarrow M^{\prime}$ which are not in the trivial homotopy class

$$
N(u, v) \leq 3(L(u)+L(v))+C(\kappa, \varrho / 20)
$$

where

$$
C(\kappa, \epsilon):=8 s h_{\kappa}^{-1}(1)+s h_{\kappa}^{-1}\left(1 / s h_{\kappa}(\epsilon)\right)
$$

with $\operatorname{sh}_{\kappa}(\epsilon):=\sinh (\sqrt{\kappa} \epsilon)$. For $u, v: G \rightarrow M$ in the trivial homotopy class, one has to assume that $M^{\prime}$ is compact and obtains in a straight forward way

$$
N(u, v) \leq \frac{1}{2}(L(u)+L(v))+\text { diameter }\left(M^{\prime}\right) .
$$




\section{Count of solutions}

Theorem 1.1 and 1.2 form the basis for a more detailed study of the set of solutions of $(0.1)$ in a given homotopy class $\zeta$ which is presented in [KL]. To simplify the exposition we state our results in the case $G=0$, i.e. for

$$
\Phi_{F}(u):=\tau(u)+F(x, u(x))=0
$$

$$
u \in \zeta
$$

For this section it is more convenient to work with Sobolev spaces. For any $j>n / 2$ we denote by $\mathcal{H}^{(j)}$ the Hilbert manifold of maps from $M$ to $M^{\prime}$ of Sobolev class $H^{j}$. By the Sobolev embedding theorem $\mathcal{H}^{(j)}$ is compactly embedded into $C^{i}\left(M, M^{\prime}\right)$ for any $0 \leq i<j-n / 2$. The connected components $\mathcal{H}_{\zeta}^{(j)}$ of the space $\mathcal{H}^{(j)}$ correspond to the homotopy classes $\zeta$ of maps in $\mathcal{H}^{(j)}$ from $M$ to $M^{\prime}$. We now fix an integer $k>2+\frac{n+n^{\prime}}{2}$, denote by $\mathcal{F}^{(k)}$ the space of $x$-dependent vector fields on $M^{\prime}$ of class $H^{k}$ and define

$$
\mathcal{M}_{\zeta}^{(k)}:=\left\{(u, F) \in \mathcal{H}_{\zeta}^{(k+2)} \times \mathcal{F}^{(k)} \mid \Phi_{F}(u)=0\right\} .
$$

We point out that Theorem 1.1 remains valid in this setting. One first observes that $\mathcal{M}_{\zeta}^{(k)}$ is a $C^{\ell}$-Hilbert manifold with $2 \leq \ell<k-\frac{n+n^{\prime}}{2}$ and that the projection $\pi_{k, \zeta}: \mathcal{M}_{\zeta}^{(k)} \mapsto \mathcal{F}^{(k)}$ is $C^{\ell}$-smooth. Let us denote by $\mathcal{F}_{\text {reg }}^{(k)}$ the set of regular values of $\pi_{k, \zeta}$. As $\pi_{k, \zeta}$ is proper, $\mathcal{F}_{r e g}^{(k)}$ is an open subset of $\mathcal{F}^{(k)}$. By the Sard-Smale theorem [Sm], it is dense in $\mathcal{F}^{(k)}$ and, for any $F \in \mathcal{F}_{r e g}^{(k)}$, the inverse image $\pi_{k, \zeta}^{-1}(F)$ is a $C^{\ell}$-submanifold of $\mathcal{M}_{\zeta}^{(k)}$. It turns out that $\pi_{k, \zeta}$ is a Fredholm map of index 0 , hence this submanifold is of dimension 0 . As $\pi_{k, \zeta}$ is proper it then follows that $\pi_{k, \zeta}^{-1}(F)$ is a finite set.

The idea is to prove that there is a natural notion of degree associated to the map $\pi_{k, \zeta}$. We follow a common approach using determinant line bundles defined for a family of Fredhom operators of some fixed index - see [CMS] for a more general framework of this construction.

Theorem 3.1 Let $k>2+\left(n+n^{\prime}\right) / 2$ and $\zeta$ be a homotopy class of maps $u: M \rightarrow M^{\prime}$ between closed Riemannian manifolds $M, M^{\prime}$ and assume that $M^{\prime}$ has a nonpositive sectional curvature. Then the projection map $\pi_{k, \zeta}: \mathcal{M}_{\zeta}^{(k)} \rightarrow \mathcal{F}^{(k)}$ admits a degree $D_{\zeta}$ which is independent of $k$. In particular, the equation $\Phi_{F}(u)=0$ has at least $\left|D_{\zeta}\right|$ solutions in $\zeta$ for any $F \in \mathcal{F}_{\text {reg }}^{(k)}$ and, in the case $D_{\zeta} \neq 0$, at least one solution for any $F \in \mathcal{F}^{(k)}$.

If in addition, the restriction of $E$ to $\zeta$ is Morse-Bott, then

$$
D_{\zeta}= \pm \chi\left(S_{0} \cap \zeta\right)
$$

where $S_{0}$ is the set of harmonic maps $u: M \rightarrow M^{\prime}$ and $\chi\left(S_{0} \cap \zeta\right)$ denotes the Euler characteristic of the submanifold $S_{0} \cap \zeta \subset C^{\infty}\left(M, M^{\prime}\right)$. 
As an example let us consider the case where $M^{\prime}$ has negative sectional curvature. In this case the restriction of the energy function $E(u)$ to any homotopy class $\zeta$ can be shown to be Morse-Bott and, according to [Ha], the set of harmonic maps $S_{0} \cap[u]$ in the homotopy class $[u]$ of a harmonic map $u$ has the property that either $S_{0} \cap[u]=\{u\}$ or $S_{0} \cap[u]$ consists of all constant maps, or $u(M)$ is a closed geodesic $\gamma$ and any other element in $S_{0} \cap[u]$ is obtained by composing $u$ with a translation along $\gamma$. Hence the integer $D_{\zeta}$ in each of the three cases is given by $1, \chi\left(M^{\prime}\right)$ or 0 respectively.

Examples show that the inequality $\sharp\left(S_{F} \cap \zeta\right) \geq\left|\chi\left(S_{0} \cap \zeta\right)\right|$ is sharp.

\section{References}

[BGS] W. Ballmann, M. Gromov, V. Schroeder: Manifolds of nonpositive curvature. Birkhäuser, Basel - Boston, 1985.

[BH] M. Bridson, A. Haefliger: Metric spaces of non-positive curvature. Springer, Berlin - New York, 1999.

[CMS] K. Cieliebak, I. Mundet i Riera, D. Salamon: Equivariant moduli problems, branched manifolds and the Euler class. ETHZ preprint, 2001.

[CS] C. Croke, V. Schroeder: The fundamental group of compact manifolds without conjugate points. Comment. Math. Helv. 61 (1986), 161 - 175.

[ES] J. Eells, J.H. Sampson: Harmonic mappings of Riemannian manifolds. Amer. J. Math. 86 (1964), p. 109 - 160.

[Gr] M. Gromov: Pseudo-holomorphic curves on almost complex manifolds. Invent. Math. 82 (1985), p. 307 - 347.

[Ha] P. Hartman: On homotopic harmonic maps. Can. J. Math. 19 (1967), p. $673-687$.

[KKS1] T. Kappeler, S. Kuksin, V. Schroeder: Perturbations of the harmonic map equation. Preprint Series, Insitute of Mathematics, University of Zurich, 2001.

[KKS2] T. Kappeler, S. Kuksin, V. Schroeder: Poincaré inequality for maps to closed manifolds of negative sectional curvature. In preparation.

[KL] T. Kappeler, J. Latschev: Counting solutions of perturbed harmonic map equations. In preparation.

[Ku] S. Kuksin: On double-periodic solutions of quasilinear Cauchy-Riemann equations. CPAM 49 (1996), p. 639 - 676.

[Sm] S. Smale: An infinite dimensional version of Sard's theorem. Amer. J. Math. 87 (1965), p. 861 - 866. 
[SY] R. Schoen, S.T. Yau: Compact group actions and the topology of manifolds with non-positive curvature. Topology 18 (1979), p. 361 - 380.

Institut Für Mathematik, Universität Zürich, Winterthurerstrasse 190, CH-8057 ZÜrich, SwitZERland.

tk@math.unizh.ch 